CRYSTALLOGRAPHIC COMMUNICATIONS

ISSN 2056-9890

Received 3 April 2015

Accepted 9 April 2015

Edited by $\mathrm{H}$. Stoeckli-Evans, University of Neuchâtel, Switzerland

Keywords: crystal structure; pyrazolone derivative; hydrogen bonding; polymer chain

CCDC reference: 1056718

Supporting information: this article has

supporting information at journals.iucr.org/e

\section{Crystal structure of (Z)- $N^{\prime}$-[1-(3-methyl-5-oxo-1- phenyl-1,5-dihydro-4H-pyrazol-4-ylidene)propyl]- benzenesulfonohydrazide}

\author{
Chuan-Chuan He and Guan-Cheng Xu*
}

Institute of Applied Chemistry, Xinjiang University, Urumqi, 830046 Xinjiang, People's Republic of China.

*Correspondence e-mail: xuguancheng@163.com

The title compound, $\mathrm{C}_{19} \mathrm{H}_{20} \mathrm{~N}_{4} \mathrm{O}_{3} \mathrm{~S}$, was synthesized by refluxing equimolar amounts of 1-phenyl-3-methyl-4-propionylpyrazol-5-one and benzenesulfonyl hydrazide in ethanol. The compound crystallizes in the keto form and the carbonyl $\mathrm{O}$ atom forms an intramolecular $\mathrm{N}-\mathrm{H} \cdots \mathrm{O}$ hydrogen bond with the neighbouring $\mathrm{NH}$ group. There is also $\mathrm{C}-\mathrm{H} \cdots \mathrm{O}$ short contact involving the neighbouring phenyl ring. Probably as a result of this, the phenyl ring is inclined to the pyrazolone ring by only $7.58(12)^{\circ}$. The dihedral angle between the phenyl ring and the benzenesulfonyl ring is $22.78(11)^{\circ}$. In the crystal, molecules are linked by pairs of $\mathrm{N}-\mathrm{H}$. . O hydrogen bonds, forming inversion dimers with an $R_{2}^{2}(14)$ ring motif. The dimers are linked via pairs of $\mathrm{C}-\mathrm{H} \cdots \mathrm{O}$ hydrogen bonds, forming chains propagating along [100].

\section{Chemical context}

Many pyrazolones and their derivatives possess biological and pharmaceutical activities, such as anticancer, antitumor and antifungal activities as well as the inhibition of lipid peroxidation (Wang et al., 1991; Yu et al., 1993; Padhyé \& Kauffman, 1985; Yang et al., 1992). Among them, the 4-acyl pyrazolone derivatives have aroused great scientific interest because of their relatively simple synthesis, wide availability and structural versatility (Raman et al., 2001; Yoshikuni, 1999; Uzoukwu et al., 1996; Yang et al., 2000).<smiles>CCC(NNS(=O)(=O)c1ccccc1)=C1C(=O)N(c2ccccc2)N=C1C</smiles>

In recent years, we have devoted our efforts to the design and synthesis of 4-acyl pyrazolone derivatives and their transition metal complexes (Zhang et al., 2004; Xu et al., 2013; Yi et al., 2014; Li et al., 2013). Such 4-acyl pyrazolone derivatives can form different types of complexes due to the multiple coordination sites and the tautomeric enol-to-keto effect. Furthermore, some of complexes have been shown to have strong antibacterial activity. For example, the copper complex $[\mathrm{Cu} L(\mathrm{EtOH})]$ [where $L$ is the $N$-(1-phenyl-3-methyl-4- 


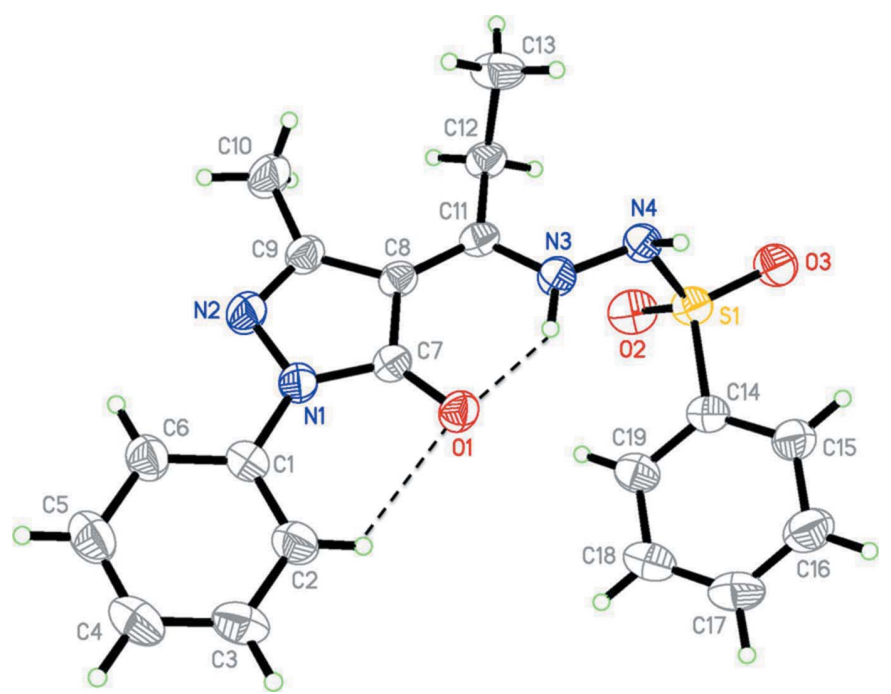

Figure 1

The molecular structure of the title compound, with the atom labelling. Displacement ellipsoids are drawn at the $30 \%$ probability level.

propenylidene-5-pyrazolone)salicylidene hydrazide anion] may be a promising drug for cancer chemotherapy (Wang et $a l ., 2007)$. This has encouraged us to investigate more 4-acyl pyrazolone derivatives and herein we report on the synthesis and crystal structure of the title compound.

\section{Structural commentary}

The molecular structure of the title compound is shown in Fig. 1. The bond lengths and angles are close to the expected values. For example, the C7-O1 bond length of 1.259 (2) $\AA$ is in good agreement with that for a $\mathrm{C}=\mathrm{O}$ double bond. The $\mathrm{C} 9-$ $\mathrm{N} 2$ bond length of 1.298 (3) $\AA$ is consistent with that for a

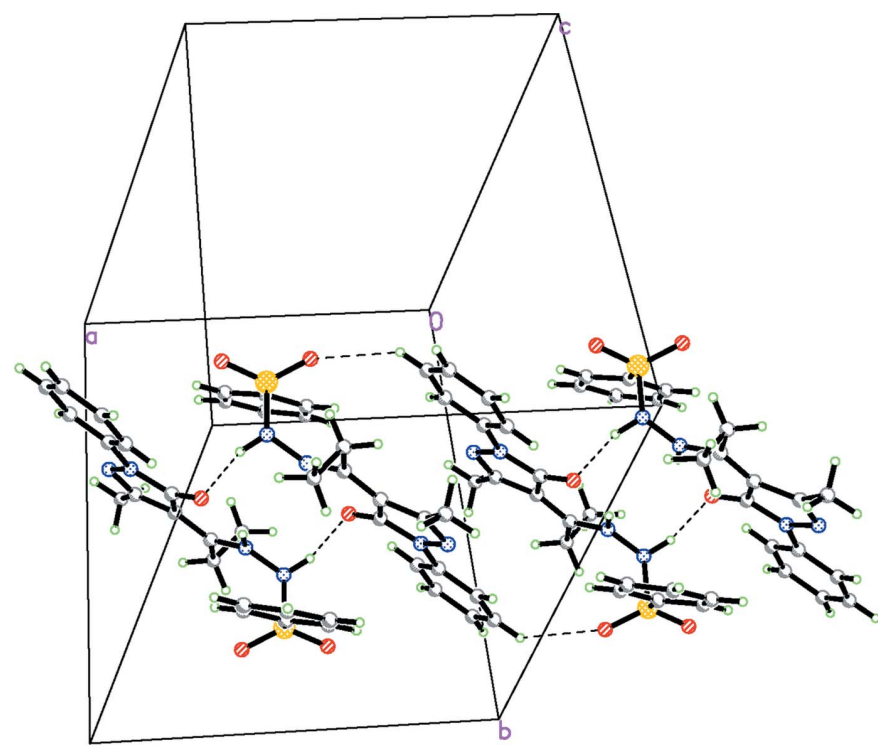

Figure 2

A view of the crystal packing of the title compound, with the hydrogen bonds shown as dashed lines (see Table 1 for details).
Table 1

Hydrogen-bond geometry $\left(\AA{ }^{\circ}\right)$.

\begin{tabular}{lllll}
\hline$D-\mathrm{H} \cdots A$ & $D-\mathrm{H}$ & $\mathrm{H} \cdots A$ & $D \cdots A$ & $D-\mathrm{H} \cdots A$ \\
\hline $\mathrm{N} 3-\mathrm{H} 3 A \cdots \mathrm{O} 1$ & 0.92 & 1.88 & $2.667(2)$ & 142 \\
$\mathrm{C} 2-\mathrm{H} 2 \cdots \mathrm{O} 1$ & 0.93 & 2.35 & $2.958(3)$ & 123 \\
$\mathrm{~N} 4-\mathrm{H} 4 A \cdots \mathrm{O} 1^{\mathrm{i}}$ & 0.93 & 1.90 & $2.800(2)$ & 162 \\
$\mathrm{C} 5-\mathrm{H} 5 \cdots \mathrm{O} 2^{\mathrm{ii}}$ & 0.93 & 2.56 & $3.299(3)$ & 137 \\
\hline
\end{tabular}

Symmetry codes: (i) $-x+2,-y+2,-z+1$; (ii) $-x+1,-y+2,-z+1$.

normal $\mathrm{C}=\mathrm{N}$ double bond, which indicates that the compound exists in the keto form. In addition, the $\mathrm{C} 11-\mathrm{N} 3$ bond length of 1.335 (2) $\AA$, is very close to that for a $\mathrm{C}-\mathrm{N}$ single bond. The $\mathrm{C} 8-\mathrm{C} 11$ bond [1.387 (3) $\AA$ ] approaches the normal $\mathrm{C}=\mathrm{C}$ bond length. These results indicate that the compound does not adopt the structure of a Schiff base.

The carbonyl $\mathrm{O}$ atom, $\mathrm{O} 1$, forms an intramolecular $\mathrm{N}-$ $\mathrm{H}$. . O hydrogen bond with the neighbouring NH group (N3$\mathrm{H} 3 A$ ), and there is a short intramolecular $\mathrm{C}-\mathrm{H} \cdots \mathrm{O}$ contact involving the neighbouring phenyl ring (C1-C6) (Table 1 and Fig. 1). This phenyl ring is inclined to the pyrazolone ring (N1/ $\mathrm{N} 2 / \mathrm{C} 7-\mathrm{C} 9)$ by $7.58(12)^{\circ}$, which is close to the value of $6.2(2)^{\circ}$ reported for a related compound, 4-isopropylidene-3-methyl1-(3-nitrophenyl)-1H-pyrazol-5(4H)-one, which also exists in the keto form (Wardell et al., 2007). The dihedral angle between the phenyl ring and the benzenesulfonyl ring (C14$\mathrm{C} 19)$ is $22.78(11)^{\circ}$. Hence, the whole molecule is non-planar, with the torsion angle about the hydrazide bond, C11 - N3N4-S1, being $-105.91(18)^{\circ}$.

Table 2

Experimental details.

\begin{tabular}{ll}
\hline Crystal data & \\
Chemical formula & $\mathrm{C}_{19} \mathrm{H}_{20} \mathrm{~N}_{4} \mathrm{O}_{3} \mathrm{~S}$ \\
$M_{\mathrm{r}}$ & 384.45 \\
Crystal system, space group & Monoclinic, $P 2_{1} / n$ \\
Temperature $(\mathrm{K})$ & 295 \\
$a, b, c(\AA)$ & $10.601(2), 16.954(3), 11.246(2)$ \\
$\beta\left({ }^{\circ}\right.$ & $107.19(3)$ \\
$V\left(\AA^{3}\right)$ & $1931.0(7)$ \\
$Z$ & 4 \\
Radiation type & Mo $K \alpha$ \\
$\mu\left(\mathrm{mm}^{-1}\right)$ & 0.19 \\
Crystal size (mm) & $0.22 \times 0.21 \times 0.20$ \\
& \\
Data collection & Rigaku R-AXIS SPIDER \\
Diffractometer & Multi-scan $(A B S C O R ;$ Higashi, \\
Absorption correction & $1995)$ \\
& $0.959,0.962$ \\
$T_{\text {min }}, T_{\text {max }}$ & $14175,3339,2574$ \\
No. of measured, independent and & \\
$\quad$ observed $[I>2 \sigma(I)]$ reflections & 0.024 \\
$R_{\text {int }}$ & 0.595 \\
(sin $\theta / \lambda)_{\text {max }}\left(\AA^{-1}\right)$ & \\
Refinement & \\
$R\left[F^{2}>2 \sigma\left(F^{2}\right)\right], w R\left(F^{2}\right), S$ & $0.040,0.131,1.08$ \\
No. of reflections & 3339 \\
No. of parameters & 247 \\
No. of restraints & 6 \\
H-atom treatment & $\mathrm{H}$-atom parameters constrained \\
$\Delta \rho_{\text {max }}, \Delta \rho_{\text {min }}\left(\mathrm{e} \AA^{-3}\right)$ & $0.19,-0.26$ \\
\hline &
\end{tabular}

Computer programs: RAPID-AUTO (Rigaku, 2004), SHELXS97, SHELXL97 and SHELXTL (Sheldrick, 2008) and PLATON (Spek, 2009). 


\section{Supramolecular features}

In the crystal, atom $\mathrm{N} 4$ acts as a donor and forms an $\mathrm{N}-$ $\mathrm{H}$... O hydrogen bond with atom $\mathrm{O} 1^{\mathrm{i}}$ (Table 1). Molecules are linked by pairs of these hydrogen bonds, forming inversion dimers with an $R_{2}^{2}(14)$ ring motif. Neighbouring dimers are linked by pairs of $\mathrm{C}-\mathrm{H}$... O hydrogen bonds, forming chains propagating along [100] (Table 1 and Fig. 2).

\section{Synthesis and crystallization}

1-Phenyl-3-methyl-4-propionyl-pyrazolone-5 (20 mmol, $4.6 \mathrm{~g}$ ) was dissolved in $25 \mathrm{~mL}$ of hot anhydrous ethanol, and an ethanol solution of benzenesulfonyl hydrazide $(20 \mathrm{mmol}$, $3.4 \mathrm{~g}$ ) was slowly added with constant stirring. After adding a few drops of glacial acetic acid as catalyst, the mixture was refluxed for $4 \mathrm{~h}$. After cooling, the precipitate that had formed was collected by filtration. A light-yellow product was obtained (yield 87\%; m.p.: 483-484 K). Yellow block-like crystals, suitable for X-ray diffraction analysis, were obtained from a methanol solution upon slow evaporation at room temperature.

\section{Refinement}

Crystal data, data collection and structure refinement details are summarized in Table 2. The $\mathrm{NH} \mathrm{H}$ atoms were located in a difference Fourier map and refined as riding atoms. C-bound $\mathrm{H}$ atoms were positioned geometrically and refined as riding: $\mathrm{C}-\mathrm{H}=0.93-0.97 \AA$ with $U_{\text {iso }}(\mathrm{H})=1.5 U_{\text {eq }}(\mathrm{C})$ for methyl $\mathrm{H}$ atoms and $1.2 U_{\mathrm{eq}}(\mathrm{N}, \mathrm{C})$ for other $\mathrm{H}$ atoms.

\section{Acknowledgements}

This work was supported by the National Science Foundation of China (No. 21161019).

\section{References}

Higashi, T. (1995). ABSCOR. Rigaku Corporation, Tokyo, Japan.

Li, H., Xu, G. C., Zhang, L., Guo, J. X. \& Jia, D. Z. (2013). Polyhedron, 55, 209-215.

Padhyé, S. \& Kauffman, G. B. (1985). Coord. Chem. Rev. 63, 127-160.

Raman, N., Kulandaisamy, A., Shunmugasundaram, A. \& Jeyasubramanian, K. (2001). Transition Met. Chem. 26, 131-135.

Rigaku (2004). RAPID-AUTO. Rigaku Corporation, Tokyo, Japan.

Sheldrick, G. M. (2008). Acta Cryst. A64, 112-122.

Spek, A. L. (2009). Acta Cryst. D65, 148-155.

Uzoukwu, B. A., Adiukwu, P. U., Al-Juaid, S. S., Hitchcock, P. B. \& Smith, J. D. (1996). Inorg. Chim. Acta, 250, 173-176.

Wang, X. H., Jia, D. Z., Liang, Y. J., Yan, S. L., Ding, Y., Chen, L. M., Shi, Z., Zeng, M. S., Liu, G. F. \& Fu, L. W. (2007). Cancer Lett. 249, 256-270.

Wang, L. F., Zhu, Y., Yang, Z. Y., Wu, J. G. \& Wang, Q. (1991). Polyhedron, 10, 2477-2461.

Wardell, J. L., Skakle, J. M. S., Low, J. N. \& Glidewell, C. (2007). Acta Cryst. C63, o462-o467.

Xu, G. C., Zhang, L., Zhang, Y. H., Guo, J. X., Shi, M. Q. \& Jia, D. Z. (2013). CrystEngComm, 15, 2873-2880.

Yang, Z. Y., Wang, L. F., Wu, J. Q. \& Li, X. Y. (1992). Chin. J. Appl. Chem. 9, 31-36.

Yang, Z. Y., Yang, R. D., Li, F. S. \& Yu, K. B. (2000). Polyhedron, 19, 2599-2604.

Yi, L. J., Xu, G. C., Zhang, L. \& Jia, D. Z. (2014). Inorg. Chem. Commun. 45, 36-39.

Yoshikuni, T. (1999). J. Mol. Catal. A Chem. 148, 285-288.

Yu, S. Y., Wang, S. X., Luo, Q. H., Wang, L. F., Peng, Z. \& Gao, X. (1993). Polyhedron, 12, 1093-1096.

Zhang, L., Liu, L., Jia, D. Z., Xu, G. C. \& Yu, K. B. (2004). Inorg. Chem. Commun. 7, 1306-1310. 


\section{supporting information}

Acta Cryst. (2015). E71, 487-489 [https://doi.org/10.1107/S2056989015007094]

\section{Crystal structure of (Z)-N'-[1-(3-methyl-5-oxo-1-phenyl-1,5-dihydro-4H- pyrazol-4-ylidene)propyl]benzenesulfonohydrazide}

\section{Chuan-Chuan He and Guan-Cheng Xu}

Computing details

Data collection: RAPID-AUTO (Rigaku, 2004); cell refinement: RAPID-AUTO (Rigaku, 2004); data reduction: RAPIDAUTO (Rigaku, 2004); program(s) used to solve structure: SHELXS97 (Sheldrick, 2008); program(s) used to refine structure: SHELXL97 (Sheldrick, 2008); molecular graphics: SHELXTL (Sheldrick, 2008); software used to prepare material for publication: SHELXL97 (Sheldrick, 2008) and PLATON (Spek, 2009).

(Z)-N'-[1-(3-Methyl-5-oxo-1-phenyl-1,5-dihydro-4H-pyrazol-4-ylidene)propyl]benzenesulfonohydrazide

\section{Crystal data}

$\mathrm{C}_{19} \mathrm{H}_{20} \mathrm{~N}_{4} \mathrm{O}_{3} \mathrm{~S}$

$M_{r}=384.45$

Monoclinic, $P 2_{1} / n$

Hall symbol: -P $2 \mathrm{yn}$

$a=10.601(2) \AA$

$b=16.954(3) \AA$

$c=11.246(2) \AA$

$\beta=107.19(3)^{\circ}$

$V=1931.0(7) \AA^{3}$

$Z=4$

\section{Data collection}

Rigaku R-AXIS SPIDER diffractometer

Radiation source: fine-focus sealed tube Graphite monochromator $\omega$ oscillation scans

Absorption correction: multi-scan

(ABSCOR; Higashi, 1995)

$T_{\min }=0.959, T_{\max }=0.962$

Refinement

Refinement on $F^{2}$

Least-squares matrix: full

$R\left[F^{2}>2 \sigma\left(F^{2}\right)\right]=0.040$

$w R\left(F^{2}\right)=0.131$

$S=1.08$

3339 reflections

247 parameters

6 restraints
$F(000)=808$

$D_{\mathrm{x}}=1.322 \mathrm{Mg} \mathrm{m}^{-3}$

Melting point: $483 \mathrm{~K}$

Mo $K \alpha$ radiation, $\lambda=0.71073 \AA$

Cell parameters from 11125 reflections

$\theta=3.1-27.5^{\circ}$

$\mu=0.19 \mathrm{~mm}^{-1}$

$T=295 \mathrm{~K}$

Block, yellow

$0.22 \times 0.21 \times 0.20 \mathrm{~mm}$

14175 measured reflections

3339 independent reflections

2574 reflections with $I>2 \sigma(I)$

$R_{\text {int }}=0.024$

$\theta_{\max }=25.0^{\circ}, \theta_{\min }=3.1^{\circ}$

$h=-12 \rightarrow 12$

$k=-20 \rightarrow 20$

$l=-13 \rightarrow 12$

Primary atom site location: structure-invariant direct methods

Secondary atom site location: difference Fourier map

Hydrogen site location: inferred from neighbouring sites

$\mathrm{H}$-atom parameters constrained

$w=1 /\left[\sigma^{2}\left(F_{\mathrm{o}}^{2}\right)+(0.0775 P)^{2}+0.2317 P\right]$

where $P=\left(F_{\mathrm{o}}{ }^{2}+2 F_{\mathrm{c}}{ }^{2}\right) / 3$ 
$(\Delta / \sigma)_{\max }=0.002$

$\Delta \rho_{\max }=0.19 \mathrm{e} \AA^{-3}$

$\Delta \rho_{\min }=-0.26 \mathrm{e} \AA^{-3}$
Extinction correction: SHELXL97 (Sheldrick, 2008), $\mathrm{Fc}^{*}=\mathrm{kFc}\left[1+0.001 \mathrm{xFc}^{2} \lambda^{3} / \sin (2 \theta)\right]^{-1 / 4}$

Extinction coefficient: $0.016(2)$

\section{Special details}

Experimental. Jacobson, $R$. (1998) Private communication

Geometry. All e.s.d.'s (except the e.s.d. in the dihedral angle between two 1.s. planes) are estimated using the full covariance matrix. The cell e.s.d.'s are taken into account individually in the estimation of e.s.d.'s in distances, angles and torsion angles; correlations between e.s.d.'s in cell parameters are only used when they are defined by crystal symmetry. An approximate (isotropic) treatment of cell e.s.d.'s is used for estimating e.s.d.'s involving 1.s. planes.

Refinement. Refinement of $F^{2}$ against ALL reflections. The weighted $R$-factor $w R$ and goodness of fit $S$ are based on $F^{2}$, conventional $R$-factors $R$ are based on $F$, with $F$ set to zero for negative $F^{2}$. The threshold expression of $F^{2}>\sigma\left(F^{2}\right)$ is used only for calculating $R$-factors(gt) etc. and is not relevant to the choice of reflections for refinement. $R$-factors based on $F^{2}$ are statistically about twice as large as those based on $F$, and $R$ - factors based on ALL data will be even larger.

Fractional atomic coordinates and isotropic or equivalent isotropic displacement parameters $\left(\hat{A}^{2}\right)$

\begin{tabular}{|c|c|c|c|c|}
\hline & $x$ & $y$ & $z$ & $U_{\text {iso }} * / U_{\text {eq }}$ \\
\hline $\mathrm{C} 1$ & $0.51056(18)$ & $0.94366(12)$ & $0.32605(18)$ & $0.0557(5)$ \\
\hline N1 & $0.60151(15)$ & $0.92936(10)$ & $0.44496(15)$ & $0.0567(4)$ \\
\hline $\mathrm{O} 1$ & $0.78565(13)$ & $1.00662(8)$ & $0.44919(12)$ & $0.0578(4)$ \\
\hline $\mathrm{S} 1$ & $1.10584(5)$ & $1.10154(3)$ & $0.79571(4)$ & $0.0588(2)$ \\
\hline $\mathrm{C} 2$ & $0.5311(2)$ & $1.00300(15)$ & $0.2494(2)$ & $0.0724(6)$ \\
\hline $\mathrm{H} 2$ & 0.6053 & 1.0352 & 0.2752 & $0.087 *$ \\
\hline N2 & $0.56682(17)$ & $0.87417(11)$ & $0.52278(17)$ & $0.0663(5)$ \\
\hline $\mathrm{O} 2$ & $1.00939(16)$ & $1.10824(10)$ & $0.85989(14)$ & $0.0776(5)$ \\
\hline $\mathrm{C} 3$ & $0.4398(3)$ & $1.01385(17)$ & $0.1336(2)$ & $0.0815(7)$ \\
\hline H3 & 0.4538 & 1.0532 & 0.0813 & $0.098^{*}$ \\
\hline N3 & $0.97144(15)$ & $0.98778(9)$ & $0.66664(14)$ & $0.0547(4)$ \\
\hline $\mathrm{H} 3 \mathrm{~A}$ & 0.9375 & 1.0074 & 0.5869 & $0.066^{*}$ \\
\hline $\mathrm{O} 3$ & $1.24221(15)$ & $1.11194(9)$ & $0.85898(14)$ & $0.0796(5)$ \\
\hline $\mathrm{C} 4$ & $0.3288(2)$ & $0.96752(17)$ & 0.0949 (2) & $0.0838(8)$ \\
\hline $\mathrm{H} 4$ & 0.2683 & 0.9752 & 0.0170 & $0.101^{*}$ \\
\hline N4 & $1.09686(15)$ & $1.00946(9)$ & $0.74215(14)$ & $0.0535(4)$ \\
\hline $\mathrm{H} 4 \mathrm{~A}$ & 1.1524 & 1.0060 & 0.6915 & $0.064 *$ \\
\hline $\mathrm{C} 5$ & $0.3086(2)$ & $0.91035(17)$ & $0.1718(2)$ & $0.0815(7)$ \\
\hline $\mathrm{H} 5$ & 0.2329 & 0.8794 & 0.1464 & $0.098^{*}$ \\
\hline C6 & $0.3989(2)$ & $0.89727(13)$ & 0.2873 & $0.0676(6)$ \\
\hline H6 & 0.3843 & 0.8574 & 0.3385 & $0.081^{*}$ \\
\hline $\mathrm{C} 7$ & $0.72508(18)$ & $0.95903(11)$ & $0.49872(17)$ & $0.0501(4)$ \\
\hline $\mathrm{C} 8$ & $0.76992(18)$ & $0.92317(11)$ & $0.61970(17)$ & $0.0503(4)$ \\
\hline C9 & $0.66454(19)$ & $0.87097(12)$ & 0.62447 (19) & $0.0589(5)$ \\
\hline $\mathrm{C} 10$ & $0.6554(2)$ & $0.81416(16)$ & $0.7229(2)$ & $0.0835(7)$ \\
\hline $\mathrm{H} 10 \mathrm{~A}$ & 0.5741 & 0.7853 & 0.6947 & $0.125^{*}$ \\
\hline H10B & 0.6578 & 0.8425 & 0.7974 & $0.125^{*}$ \\
\hline $\mathrm{H} 10 \mathrm{C}$ & 0.7284 & 0.7781 & 0.7399 & $0.125^{*}$ \\
\hline $\mathrm{C} 11$ & $0.89157(18)$ & $0.93953(11)$ & $0.70462(16)$ & 0.0493 (4) \\
\hline $\mathrm{C} 12$ & $0.9392(2)$ & $0.90616(12)$ & $0.83395(18)$ & $0.0600(5)$ \\
\hline $\mathrm{H} 12 \mathrm{~A}$ & 0.8637 & 0.8913 & 0.8610 & $0.072 *$ \\
\hline
\end{tabular}




$\begin{array}{lllll}\text { H12B } & 0.9879 & 0.9464 & 0.8903 & 0.072^{*} \\ \text { C13 } & 1.0270(3) & 0.83473(15) & 0.8404(2) & 0.0869(8) \\ \text { H13A } & 0.9812 & 0.7962 & 0.7806 & 0.130^{*} \\ \text { H13B } & 1.0492 & 0.8124 & 0.9224 & 0.130^{*} \\ \text { H13C } & 1.1064 & 0.8504 & 0.8222 & 0.130^{*} \\ \text { C14 } & 1.06098(19) & 1.16434(11) & 0.66519(18) & 0.0568(5) \\ \text { C15 } & 1.1583(2) & 1.20180(14) & 0.6279(2) & 0.0734(6) \\ \text { H15 } & 1.2470 & 1.1931 & 0.6698 & 0.088^{*} \\ \text { C16 } & 1.1223(3) & 1.25235(15) & 0.5273(2) & 0.0871(7) \\ \text { H16 } & 1.1871 & 1.2779 & 0.5012 & 0.104^{*} \\ \text { C17 } & 0.9924(3) & 1.26498(15) & 0.4660(2) & 0.0838(7) \\ \text { H17 } & 0.9691 & 1.3005 & 0.4001 & 0.101^{*} \\ \text { C18 } & 0.8952(3) & 1.22547(15) & 0.5008(2) & 0.0790(6) \\ \text { H18 } & 0.8068 & 1.2326 & 0.4564 & 0.095^{*} \\ \text { C19 } & 0.9296(2) & 1.17543(13) & 0.60173(19) & 0.0655(6) \\ \text { H19 } & 0.8646 & 1.1493 & 0.6268 & 0.079^{*}\end{array}$

Atomic displacement parameters $\left(\AA^{2}\right)$

\begin{tabular}{|c|c|c|c|c|c|c|}
\hline & $U^{11}$ & $U^{22}$ & $U^{33}$ & $U^{12}$ & $U^{13}$ & $U^{23}$ \\
\hline $\mathrm{C} 1$ & $0.0531(11)$ & $0.0625(12)$ & $0.0501(11)$ & $0.0071(8)$ & $0.0130(8)$ & $-0.0009(9)$ \\
\hline N1 & $0.0555(9)$ & $0.0618(10)$ & $0.0502(9)$ & $-0.0073(7)$ & $0.0115(7)$ & $0.0068(8)$ \\
\hline $\mathrm{O} 1$ & $0.0642(8)$ & $0.0615(8)$ & $0.0462(7)$ & $-0.0117(6)$ & $0.0141(6)$ & $0.0059(6)$ \\
\hline S1 & $0.0705(4)$ & $0.0586(4)$ & $0.0406(3)$ & $-0.0030(2)$ & $0.0062(2)$ & $-0.0046(2)$ \\
\hline $\mathrm{C} 2$ & $0.0652(13)$ & $0.0841(16)$ & $0.0624(14)$ & $0.0019(11)$ & $0.0100(10)$ & $0.0130(12)$ \\
\hline N2 & $0.0668(11)$ & $0.0688(12)$ & $0.0632(11)$ & $-0.0128(8)$ & $0.0187(9)$ & $0.0121(9)$ \\
\hline $\mathrm{O} 2$ & $0.1026(11)$ & $0.0837(11)$ & $0.0520(9)$ & $0.0062(8)$ & $0.0312(8)$ & $-0.0069(8)$ \\
\hline $\mathrm{C} 3$ & $0.0821(17)$ & $0.0970(19)$ & $0.0604(14)$ & $0.0190(13)$ & $0.0132(12)$ & $0.0191(13)$ \\
\hline N3 & $0.0611(10)$ & $0.0595(10)$ & $0.0388(8)$ & $-0.0095(7)$ & $0.0075(7)$ & $0.0013(7)$ \\
\hline $\mathrm{O} 3$ & $0.0780(8)$ & $0.0758(10)$ & $0.0623(9)$ & $-0.0147(7)$ & $-0.0146(7)$ & $-0.0001(7)$ \\
\hline $\mathrm{C} 4$ & $0.0763(16)$ & $0.100(2)$ & $0.0614(15)$ & $0.0200(14)$ & $-0.0012(12)$ & $-0.0071(14)$ \\
\hline N4 & $0.0558(9)$ & $0.0574(10)$ & $0.0437(9)$ & $-0.0033(7)$ & $0.0091(7)$ & $-0.0008(7)$ \\
\hline $\mathrm{C} 5$ & $0.0689(15)$ & $0.0896(18)$ & $0.0735(16)$ & $0.0018(12)$ & $0.0019(12)$ & $-0.0150(14)$ \\
\hline C6 & $0.0627(13)$ & $0.0680(14)$ & $0.0670(14)$ & $-0.0012(10)$ & $0.0112(10)$ & $-0.0069(11)$ \\
\hline $\mathrm{C} 7$ & $0.0576(11)$ & $0.0464(10)$ & $0.0469(11)$ & $-0.0025(8)$ & $0.0162(8)$ & $-0.0002(8)$ \\
\hline $\mathrm{C} 8$ & $0.0609(11)$ & $0.0474(10)$ & $0.0427(10)$ & $-0.0016(8)$ & $0.0156(8)$ & $0.0021(8)$ \\
\hline C9 & $0.0655(12)$ & $0.0557(12)$ & $0.0559(12)$ & $-0.0068(9)$ & $0.0185(10)$ & $0.0068(9)$ \\
\hline $\mathrm{C} 10$ & $0.0920(17)$ & $0.0807(16)$ & $0.0752(16)$ & $-0.0210(12)$ & $0.0207(13)$ & $0.0245(13)$ \\
\hline C11 & $0.0635(11)$ & $0.0435(10)$ & $0.0409(10)$ & $0.0012(8)$ & $0.0154(8)$ & $0.0002(8)$ \\
\hline $\mathrm{C} 12$ & $0.0749(13)$ & $0.0577(12)$ & $0.0436(11)$ & $-0.0016(9)$ & $0.0116(9)$ & $0.0051(9)$ \\
\hline $\mathrm{C} 13$ & $0.1057(19)$ & $0.0747(17)$ & $0.0699(16)$ & $0.0203(13)$ & $0.0099(13)$ & $0.0182(12)$ \\
\hline C14 & $0.0694(13)$ & $0.0503(11)$ & $0.0460(11)$ & $0.0037(9)$ & $0.0099(9)$ & $-0.0067(8)$ \\
\hline $\mathrm{C} 15$ & $0.0750(14)$ & $0.0680(14)$ & $0.0705(14)$ & $-0.0041(11)$ & $0.0113(11)$ & $0.0089(12)$ \\
\hline $\mathrm{C} 16$ & 0.1027 (19) & $0.0792(17)$ & $0.0794(17)$ & $-0.0031(14)$ & $0.0272(15)$ & $0.0186(14)$ \\
\hline C17 & 0.1109 (19) & $0.0750(16)$ & $0.0588(14)$ & $0.0157(12)$ & 0.0145 & $0.0111(12)$ \\
\hline $\mathrm{C} 18$ & $0.0837(15)$ & $0.0764(16)$ & $0.0664(14)$ & $0.0237(11)$ & 0.0060 & $0.0025(12)$ \\
\hline C19 & $0.0697(13)$ & $0.0644(13)$ & $0.0600(13)$ & $0.0122(10)$ & $0.0155(10)$ & $-0.0039(10)$ \\
\hline
\end{tabular}


Geometric parameters $\left(\AA,{ }^{\circ}\right)$

\begin{tabular}{|c|c|c|c|}
\hline $\mathrm{C} 1-\mathrm{C} 6$ & $1.380(3)$ & $\mathrm{C} 8-\mathrm{C} 11$ & $1.387(3)$ \\
\hline $\mathrm{C} 1-\mathrm{C} 2$ & $1.383(3)$ & $\mathrm{C} 8-\mathrm{C} 9$ & $1.439(3)$ \\
\hline $\mathrm{C} 1-\mathrm{N} 1$ & $1.419(2)$ & $\mathrm{C} 9-\mathrm{C} 10$ & $1.492(3)$ \\
\hline $\mathrm{N} 1-\mathrm{C} 7$ & $1.366(2)$ & $\mathrm{C} 10-\mathrm{H} 10 \mathrm{~A}$ & 0.9600 \\
\hline $\mathrm{N} 1-\mathrm{N} 2$ & $1.402(2)$ & $\mathrm{C} 10-\mathrm{H} 10 \mathrm{~B}$ & 0.9600 \\
\hline $\mathrm{O} 1-\mathrm{C} 7$ & $1.259(2)$ & $\mathrm{C} 10-\mathrm{H} 10 \mathrm{C}$ & 0.9600 \\
\hline $\mathrm{S} 1-\mathrm{O} 2$ & $1.4197(17)$ & $\mathrm{C} 11-\mathrm{C} 12$ & $1.502(3)$ \\
\hline $\mathrm{S} 1-\mathrm{O} 3$ & $1.4211(16)$ & $\mathrm{C} 12-\mathrm{C} 13$ & $1.517(3)$ \\
\hline $\mathrm{S} 1-\mathrm{N} 4$ & $1.6658(17)$ & $\mathrm{C} 12-\mathrm{H} 12 \mathrm{~A}$ & 0.9700 \\
\hline $\mathrm{S} 1-\mathrm{C} 14$ & $1.761(2)$ & $\mathrm{C} 12-\mathrm{H} 12 \mathrm{~B}$ & 0.9700 \\
\hline $\mathrm{C} 2-\mathrm{C} 3$ & $1.386(3)$ & $\mathrm{C} 13-\mathrm{H} 13 \mathrm{~A}$ & 0.9600 \\
\hline $\mathrm{C} 2-\mathrm{H} 2$ & 0.9300 & C13-H13B & 0.9600 \\
\hline $\mathrm{N} 2-\mathrm{C} 9$ & $1.298(3)$ & $\mathrm{C} 13-\mathrm{H} 13 \mathrm{C}$ & 0.9600 \\
\hline $\mathrm{C} 3-\mathrm{C} 4$ & $1.374(4)$ & $\mathrm{C} 14-\mathrm{C} 19$ & $1.378(3)$ \\
\hline $\mathrm{C} 3-\mathrm{H} 3$ & 0.9300 & $\mathrm{C} 14-\mathrm{C} 15$ & $1.379(3)$ \\
\hline $\mathrm{N} 3-\mathrm{C} 11$ & $1.335(2)$ & $\mathrm{C} 15-\mathrm{C} 16$ & $1.380(3)$ \\
\hline $\mathrm{N} 3-\mathrm{N} 4$ & $1.399(2)$ & $\mathrm{C} 15-\mathrm{H} 15$ & 0.9300 \\
\hline N3-H3A & 0.9241 & $\mathrm{C} 16-\mathrm{C} 17$ & $1.362(3)$ \\
\hline $\mathrm{C} 4-\mathrm{C} 5$ & $1.357(4)$ & $\mathrm{C} 16-\mathrm{H} 16$ & 0.9300 \\
\hline $\mathrm{C} 4-\mathrm{H} 4$ & 0.9300 & $\mathrm{C} 17-\mathrm{C} 18$ & $1.380(4)$ \\
\hline $\mathrm{N} 4-\mathrm{H} 4 \mathrm{~A}$ & 0.9335 & C17-H17 & 0.9300 \\
\hline $\mathrm{C} 5-\mathrm{C} 6$ & $1.385(3)$ & $\mathrm{C} 18-\mathrm{C} 19$ & $1.377(3)$ \\
\hline $\mathrm{C} 5-\mathrm{H} 5$ & 0.9300 & C18-H18 & 0.9300 \\
\hline $\mathrm{C} 6-\mathrm{H} 6$ & 0.9300 & C19-H19 & 0.9300 \\
\hline $\mathrm{C} 7-\mathrm{C} 8$ & $1.437(3)$ & & \\
\hline $\mathrm{C} 6-\mathrm{C} 1-\mathrm{C} 2$ & $119.73(19)$ & $\mathrm{N} 2-\mathrm{C} 9-\mathrm{C} 10$ & $118.08(18)$ \\
\hline $\mathrm{C} 6-\mathrm{C} 1-\mathrm{N} 1$ & $118.73(19)$ & $\mathrm{C} 8-\mathrm{C} 9-\mathrm{C} 10$ & $130.27(18)$ \\
\hline $\mathrm{C} 2-\mathrm{C} 1-\mathrm{N} 1$ & $121.54(18)$ & $\mathrm{C} 9-\mathrm{C} 10-\mathrm{H} 10 \mathrm{~A}$ & 109.5 \\
\hline $\mathrm{C} 7-\mathrm{N} 1-\mathrm{N} 2$ & $111.31(15)$ & $\mathrm{C} 9-\mathrm{C} 10-\mathrm{H} 10 \mathrm{~B}$ & 109.5 \\
\hline $\mathrm{C} 7-\mathrm{N} 1-\mathrm{C} 1$ & $130.51(17)$ & $\mathrm{H} 10 \mathrm{~A}-\mathrm{C} 10-\mathrm{H} 10 \mathrm{~B}$ & 109.5 \\
\hline $\mathrm{N} 2-\mathrm{N} 1-\mathrm{C} 1$ & $118.13(15)$ & $\mathrm{C} 9-\mathrm{C} 10-\mathrm{H} 10 \mathrm{C}$ & 109.5 \\
\hline $\mathrm{O} 2-\mathrm{S} 1-\mathrm{O} 3$ & $120.96(10)$ & $\mathrm{H} 10 \mathrm{~A}-\mathrm{C} 10-\mathrm{H} 10 \mathrm{C}$ & 109.5 \\
\hline $\mathrm{O} 2-\mathrm{S} 1-\mathrm{N} 4$ & $106.62(9)$ & $\mathrm{H} 10 \mathrm{~B}-\mathrm{C} 10-\mathrm{H} 10 \mathrm{C}$ & 109.5 \\
\hline $\mathrm{O} 3-\mathrm{S} 1-\mathrm{N} 4$ & $103.79(9)$ & $\mathrm{N} 3-\mathrm{C} 11-\mathrm{C} 8$ & $116.80(16)$ \\
\hline $\mathrm{O} 2-\mathrm{S} 1-\mathrm{C} 14$ & $108.53(10)$ & $\mathrm{N} 3-\mathrm{C} 11-\mathrm{C} 12$ & $118.51(16)$ \\
\hline $\mathrm{O} 3-\mathrm{S} 1-\mathrm{C} 14$ & $109.05(10)$ & $\mathrm{C} 8-\mathrm{C} 11-\mathrm{C} 12$ & $124.69(17)$ \\
\hline $\mathrm{N} 4-\mathrm{S} 1-\mathrm{C} 14$ & $107.02(8)$ & $\mathrm{C} 11-\mathrm{C} 12-\mathrm{C} 13$ & $111.87(18)$ \\
\hline $\mathrm{C} 1-\mathrm{C} 2-\mathrm{C} 3$ & $119.1(2)$ & $\mathrm{C} 11-\mathrm{C} 12-\mathrm{H} 12 \mathrm{~A}$ & 109.2 \\
\hline $\mathrm{C} 1-\mathrm{C} 2-\mathrm{H} 2$ & 120.4 & $\mathrm{C} 13-\mathrm{C} 12-\mathrm{H} 12 \mathrm{~A}$ & 109.2 \\
\hline $\mathrm{C} 3-\mathrm{C} 2-\mathrm{H} 2$ & 120.4 & $\mathrm{C} 11-\mathrm{C} 12-\mathrm{H} 12 \mathrm{~B}$ & 109.2 \\
\hline $\mathrm{C} 9-\mathrm{N} 2-\mathrm{N} 1$ & $106.83(16)$ & $\mathrm{C} 13-\mathrm{C} 12-\mathrm{H} 12 \mathrm{~B}$ & 109.2 \\
\hline $\mathrm{C} 4-\mathrm{C} 3-\mathrm{C} 2$ & $121.1(3)$ & $\mathrm{H} 12 \mathrm{~A}-\mathrm{C} 12-\mathrm{H} 12 \mathrm{~B}$ & 107.9 \\
\hline $\mathrm{C} 4-\mathrm{C} 3-\mathrm{H} 3$ & 119.4 & $\mathrm{C} 12-\mathrm{C} 13-\mathrm{H} 13 \mathrm{~A}$ & 109.5 \\
\hline $\mathrm{C} 2-\mathrm{C} 3-\mathrm{H} 3$ & 119.4 & $\mathrm{C} 12-\mathrm{C} 13-\mathrm{H} 13 \mathrm{~B}$ & 109.5 \\
\hline $\mathrm{C} 11-\mathrm{N} 3-\mathrm{N} 4$ & $123.07(15)$ & $\mathrm{H} 13 \mathrm{~A}-\mathrm{C} 13-\mathrm{H} 13 \mathrm{~B}$ & 109.5 \\
\hline
\end{tabular}




$\mathrm{C} 11-\mathrm{N} 3-\mathrm{H} 3 \mathrm{~A}$
$\mathrm{~N} 4-\mathrm{N} 3-\mathrm{H} 3 \mathrm{~A}$
$\mathrm{C} 5-\mathrm{C} 4-\mathrm{C} 3$
$\mathrm{C} 5-\mathrm{C} 4-\mathrm{H} 4$
$\mathrm{C} 3-\mathrm{C} 4-\mathrm{H} 4$
$\mathrm{~N} 3-\mathrm{N} 4-\mathrm{S} 1$
$\mathrm{~N} 3-\mathrm{N} 4-\mathrm{H} 4 \mathrm{~A}$
$\mathrm{~S} 1-\mathrm{N} 4-\mathrm{H} 4 \mathrm{~A}$
$\mathrm{C} 4-\mathrm{C} 5-\mathrm{C} 6$
$\mathrm{C} 4-\mathrm{C} 5-\mathrm{H} 5$
$\mathrm{C} 6-\mathrm{C} 5-\mathrm{H} 5$
$\mathrm{C} 1-\mathrm{C} 6-\mathrm{C} 5$
$\mathrm{C} 1-\mathrm{C} 6-\mathrm{H} 6$
$\mathrm{C} 5-\mathrm{C} 6-\mathrm{H} 6$
$\mathrm{O} 1-\mathrm{C} 7-\mathrm{N} 1$
$\mathrm{O} 1-\mathrm{C} 7-\mathrm{C} 8$
N1-C7-C8
$\mathrm{C} 11-\mathrm{C} 8-\mathrm{C} 7$
$\mathrm{C} 11-\mathrm{C} 8-\mathrm{C} 9$
$\mathrm{C} 7-\mathrm{C} 8-\mathrm{C} 9$
N2-C $9-\mathrm{C} 8$

115.4

121.5

$119.2(2)$

120.4

120.4

$114.10(12)$

105.7

107.5

$121.1(2)$

119.5

119.5

119.7 (2)

120.2

120.2

$126.01(17)$

$128.38(17)$

$105.59(16)$

$123.18(17)$

$132.19(17)$

$104.62(16)$

$111.59(18)$
$\mathrm{C} 12-\mathrm{C} 13-\mathrm{H} 13 \mathrm{C}$

$\mathrm{H} 13 \mathrm{~A}-\mathrm{C} 13-\mathrm{H} 13 \mathrm{C}$

$\mathrm{H} 13 \mathrm{~B}-\mathrm{C} 13-\mathrm{H} 13 \mathrm{C}$

C19-C14-C15

$\mathrm{C} 19-\mathrm{C} 14-\mathrm{S} 1$

$\mathrm{C} 15-\mathrm{C} 14-\mathrm{S} 1$

$\mathrm{C} 14-\mathrm{C} 15-\mathrm{C} 16$

C14-C15-H15

C16-C15-H15

$\mathrm{C} 17-\mathrm{C} 16-\mathrm{C} 15$

$\mathrm{C} 17-\mathrm{C} 16-\mathrm{H} 16$

$\mathrm{C} 15-\mathrm{C} 16-\mathrm{H} 16$

$\mathrm{C} 16-\mathrm{C} 17-\mathrm{C} 18$

$\mathrm{C} 16-\mathrm{C} 17-\mathrm{H} 17$

C18-C17-H17

$\mathrm{C} 19-\mathrm{C} 18-\mathrm{C} 17$

C19-C18-H18

C17-C18-H18

C18-C19-C14

C18-C19-H19

C14-C19-H19
109.5

109.5

109.5

120.9 (2)

$119.79(17)$

$119.35(16)$

$119.0(2)$

120.5

120.5

120.4 (2)

119.8

119.8

120.5 (2)

119.7

119.7

119.7 (2)

120.2

120.2

119.5 (2)

120.3

120.3

Hydrogen-bond geometry $\left(A,{ }^{\circ}\right)$

\begin{tabular}{lllll}
\hline$D-\mathrm{H} \cdots A$ & $D-\mathrm{H}$ & $\mathrm{H} \cdots A$ & $D \cdots A$ & $D-\mathrm{H} \cdots A$ \\
\hline $\mathrm{N} 3-\mathrm{H} 3 A \cdots \mathrm{O} 1$ & 0.92 & 1.88 & $2.667(2)$ & 142 \\
$\mathrm{C} 2-\mathrm{H} 2 \cdots \mathrm{O} 1$ & 0.93 & 2.35 & $2.958(3)$ & 123 \\
$\mathrm{~N} 4-\mathrm{H} 4 A \cdots \mathrm{O} 1^{\mathrm{i}}$ & 0.93 & 1.90 & $2.800(2)$ & 162 \\
$\mathrm{C} 5-\mathrm{H} 5 \cdots \mathrm{O} 2^{\mathrm{ii}}$ & 0.93 & 2.56 & $3.299(3)$ & 137
\end{tabular}

Symmetry codes: (i) $-x+2,-y+2,-z+1$; (ii) $-x+1,-y+2,-z+1$. 